\title{
Leray-Schauder-type fixed point theorems in Banach algebras and application to quadratic integral equations
}

\author{
Abdelmjid Khchine ${ }^{1}$, Lahcen Maniar ${ }^{1}$ and Mohamed-Aziz Taoudi ${ }^{*}$
}

\footnotetext{
"Correspondence: a.taoudi@uca.ma

${ }^{2}$ National School of Applied

Sciences, Cadi Ayyad University,

Marrakesh, Morocco

Full list of author information is

available at the end of the article
}

\begin{abstract}
In this paper, we present new fixed point theorems in Banach algebras relative to the weak topology. Our fixed point results are obtained under Leray-Schauder-type boundary conditions. These results improve and complement a number of earlier works. As an application, we establish some existence results for a broad class of quadratic integral equations.
\end{abstract}

MSC: $47 \mathrm{H} 10 ; 45 \mathrm{G} 10$

Keywords: fixed point theorem; Banach algebra; quadratic integral equation; measure of weak noncompactness; weakly condensing

\section{Introduction}

The need for a fixed point theory in Banach algebras arose out of the study of quadratic integral equations. Those equations have received increasing attention during recent years due to their applications in diverse fields of science and engineering, for example, the theory of radiative transfer [1], statistical mechanics [2], biomathematics [3], signal theory [4], kinetic theory of gases [5], transport theory [6], and so on.

The problem of existence of solution to quadratic integral equations may be usually reduced to a fixed point problem of the form

$$
A x B x+C x=x, \quad x \in \Omega,
$$

where $\Omega$ is a nonempty subset of a Banach algebra $X$.

In recent years, significant advances have been made in the development of fixed point theory in Banach algebras using the norm topology and applications to quadratic integral equations. We quote the contributions by Leggett [7], Majorana and Marano [8], Banas and Lecko [9], Dhage [10-14], and many others (see, e.g., [15-18] and the references therein).

However, only a few papers have been up to now devoted to the existence of fixed points for mappings acting on Banach algebras relative to the weak topology [19-21].

One of the most important results for solving (1.1) using the norm topology is the following result due to Dhage [12]. This result can be seen as an analogue of Krasnosel'skii's fixed point theorem in Banach algebras.

(c) 2016 Khchine et al. This article is distributed under the terms of the Creative Commons Attribution 4.0 International License (http://creativecommons.org/licenses/by/4.0/), which permits unrestricted use, distribution, and reproduction in any medium, provided you give appropriate credit to the original author(s) and the source, provide a link to the Creative Commons license, and indicate if changes were made. 
Theorem 1.1 Let $\Omega$ be a nonempty bounded closed convex subset of a Banach algebra X.

Let $B: \Omega \rightarrow X$ and $A, C: X \rightarrow X$ be continuous operators satisfying:

(i) B is completely continuous,

(ii) $A$ and $C$ are Lipschitzian mappings with constants $k_{A}$ and $k_{C}$, respectively,

(iii) the equality $x=A x B y+C x$ with $y \in \Omega$ implies $x \in \Omega$.

Then the operator equation $x=A x B x+C x$ has a solution whenever $Q k_{A}+k_{C}<1$, where $Q=\|B(\Omega)\|$.

Very recently, Banas and Taoudi [19] proved the following analogue of Theorem 1.1 for the weak topology.

Theorem 1.2 Let $\Omega$ be a nonempty closed and convex subset of a Banach algebra $X$ with property $(\mathcal{P})$. Let $B: \Omega \rightarrow X$ and $A, C: X \rightarrow X$ be weakly sequentially continuous operators satisfying:

(i) $B(\Omega)$ is relatively weakly compact,

(ii) $A$ and $C$ are Lipschitzian mappings with constants $k_{A}$ and $k_{C}$, respectively,

(iii) the equality $x=A x B y+C x$ with $y \in \Omega$ implies $x \in \Omega$.

Then the operator equation $x=A x B x+C x$ has a solution whenever $Q k_{A}+k_{C}<1$, where $Q=\|B(\Omega)\|$.

Recall that a Banach algebra $X$ is said to have property $(\mathcal{P})$ if for every two sequences $\left\{x_{n}\right\}$ and $\left\{y_{n}\right\}$ in $X$ such that $x_{n} \rightarrow x$ and $y_{n} \rightarrow y$ for some $x, y \in X$, we have $x_{n} y_{n} \rightarrow x y$.

We emphasize that Theorems 1.1 and 1.2 are stated under the Burton-type boundary condition that

$$
(x=A x B y+C x, y \in \Omega) \quad \text { implies } \quad x \in \Omega .
$$

In some applications, it is difficult and sometimes impossible to construct a set $\Omega$ so that (1.2) holds. This entails the importance of considering other 'boundary conditions,', namely Leray-Schauder-type conditions [14], Furi-Pera-type conditions [18], and many others.

The aim of this paper is to establish new fixed point theorems in Banach algebras relative to the weak topology under Leray-Schauder-type boundary conditions. Then, we replace the weak continuity of the involved operators by the concepts of ww-compactness and ws-compactness and interchange the weak compactness by some alternative conditions formulated using the axiomatic measure of weak noncompactness. Our results improve and encompass several earlier related works. As an application, we prove the existence of a continuous solution for a broad class of quadratic integral equations.

This paper is arranged as follows. In Section 1, we fix the notation and present some key tools that will be used to prove our main results. In Section 2, we prove some fixed point results in Banach algebras under boundary conditions of Leray-Schauder type. Finally, in the last section, we use some materials from previous sections to develop an existence theory for a broad class of quadratic integral equations. For the remainder of this section, we gather some notations and preliminary facts. Let $X$ be a Banach algebra with norm $\|\cdot\|$, and let $X^{*}$ denote the topological dual of $X$. We use the symbol $B(x, r)$ to denote the closed ball centered at $x$ with radius $r$. We write $B_{r}$ to denote $B(0, r)$. Further, we denote by $B(X)$ the family of all nonempty and bounded subsets of $X$. The symbol $\mathcal{K}^{w}(X)$ stands 
for the family of all weakly compact subsets of $X$. For any bounded subset $\Omega$ of $X$, we put $\|\Omega\|=\sup \{\|x\|: x \in \Omega\}$.

In the sequel, we will adopt the following definition of the axiomatic measure of weak noncompactness [22].

Definition 1.3 A function $\psi: B(X) \longrightarrow \mathbb{R}^{+}$is said to be a measure of weak noncompactness if it satisfies the following conditions:

(i) The family $\operatorname{ker} \psi=\{M \in B(X): \psi(M)=0\}$ is nonempty, and $\operatorname{ker} \psi$ is contained in the set of relatively weakly compact subsets of $X$,

(ii) $M_{1} \subset M_{2} \Longrightarrow \psi\left(M_{1}\right) \leq \psi\left(M_{2}\right)$,

(iii) $\psi(c o(M))=\psi(M)$, where $c o(M)$ is the convex hull of $M$,

(iv) $\psi\left(\lambda M_{1}+(1-\lambda) M_{2}\right) \leq \lambda \psi\left(M_{1}\right)+(1-\lambda) \psi\left(M_{2}\right)$ for $\lambda \in[0,1]$,

(v) if $\left(M_{n}\right)_{n \geq 1}$ is a sequence of nonempty, weakly closed subsets of $X$ with $M_{1}$ bounded and $M_{1} \supseteq M_{2} \supseteq \cdots \supseteq M_{n} \supseteq \cdots$ such that $\lim _{n \rightarrow+\infty} \psi\left(M_{n}\right)=0$, then the set $M_{\infty}:=\bigcap_{n=1}^{\infty} M_{n}$ is nonempty.

Notice that the set $M_{\infty}$ belongs to $\operatorname{ker} \psi$ since $\psi\left(M_{\infty}\right) \leq \psi\left(M_{n}\right)$ for every $n$ and $\lim _{n \rightarrow+\infty} \psi\left(M_{n}\right)=0$. Also, we can readily check that the measure $\psi$ satisfies, for all $M \in B(X)$,

$$
\psi\left(\overline{M^{w}}\right)=\psi(M)
$$

We say that $\psi$ is nonsingular if

$$
\psi\left(M \cup\left\{x_{0}\right\}\right)=\psi(M)
$$

for each $x_{0} \in X$.

The first important example of a measure of weak noncompactness was defined by De Blasi [23] as follows:

$$
w(M)=\inf \left\{r>0: \text { there exists } W \in \mathcal{K}^{w}(X) \text { with } M \subset W+B_{r}\right\}
$$

for all $M \in B(X)$. Notice that the De Blasi measure of weak noncompactness $w$ is nonsingular.

We summarize some useful properties of $w$ in the following proposition. For a proof, we refer the reader to [23].

Proposition 1.4 For all bounded subsets $M_{1}$ and $M_{2}$ of $X$, we have:

(a) $w\left(M_{1}\right)=0$ if and only if $M_{1}$ is relatively weakly compact,

(b) $w\left(M_{1}+M_{2}\right) \leq w\left(M_{1}\right)+w\left(M_{2}\right)$,

(c) $w\left(M_{1} \cup M_{2}\right)=\max \left(w\left(M_{1}\right), w\left(M_{2}\right)\right)$.

Definition 1.5 [20, 24] Let $\Omega$ be a nonempty subset of a Banach space $X$, and $\psi$ be a measure of weak noncompactness on $X$. Let $T: \Omega \rightarrow X$ be a mapping. We say that:

(i) $T$ is weakly sequentially continuous if it maps weakly convergent sequences into weakly convergent sequences, 
(ii) $T$ is $\psi$-condensing if $T$ is bounded (i.e., it takes bounded sets into bounded sets) and $\psi(T(M))<\psi(M)$ for any bounded subset $M$ of $\Omega$ with $\psi(M)>0$,

(iii) $T$ is weakly compact if $T(\Omega)$ is relatively weakly compact,

(iv) $T$ is $w s$ - compact if it is continuous and for any weakly convergent sequence $\left(x_{n}\right)$ in $\Omega$, the sequence $\left(T x_{n}\right)$ has a strongly convergent subsequence in $X$,

(v) $T$ is $w w$-compact if it is continuous and for any weakly convergent sequence $\left(x_{n}\right)$ in $\Omega$, the sequence $\left(T x_{n}\right)$ has a weakly convergent subsequence in $X$.

Notice that the concepts of $w w$-compact and ws-compact mappings arise naturally in the study of integral and partial differential equations (see [24-32]). It is worth noting that $w w-$ compact and ws-compact mappings are not necessarily weakly sequentially continuous [25].

The following result is crucial for our purpose.

Theorem 1.6 ([33], Theorem 1.1) Let X be a Banach space.

(i) Let $H$ be a bounded subset of $C([0, T], X)$. Then

$$
\sup _{t \in[0, T]} w(H(t)) \leq w(H)
$$

where $H(t)=\{x(t) ; x \in H\}$;

(ii) Let $H \subset C([0, T], X)$ be bounded and equicontinuous. Then

$$
w(H)=\sup _{t \in[0, T]} w(H(t))=w(H[0, T]),
$$

where $H[0, T]=\bigcup_{t \in[0, T]} H(t)$.

For later use, we recall the mean value theorem for the Pettis integral; see [34], Theorem 3.

Lemma 1.7 Let $X$ be a Banach space, and $h:[a, b] \longrightarrow X$ be a Pettis-integrable function. Then

$$
\int_{a}^{b} h(s) d s \in(b-a) \overline{c o}(h[a, b]) .
$$

The following lemma is well known.

Lemma 1.8 ([35], Lemma 2.8) Let $X$ be a Banach space, and $F: X \longrightarrow X$ be a Lipschitzian mapping with constant $k$. If, moreover, $F$ is ww-compact, then for any bounded subset $M$ of $X$, we have $w(F(M)) \leq k w(M)$.

Definition 1.9 [19] Let $X$ be a Banach algebra. We say that $X$ is a WC-Banach algebra if the product $K \cdot K^{\prime}$ of arbitrary weakly compact subsets $K$ and $K^{\prime}$ of $X$ is weakly compact.

Lemma 1.10 [19] Let $M$ and $M^{\prime}$ be bounded subsets of a WC-Banach algebra $X$. Then $w\left(M \cdot M^{\prime}\right) \leq\left\|M^{\prime}\right\| w(M)+\|M\| w\left(M^{\prime}\right)+w(M) w\left(M^{\prime}\right)$.

In particular, if $M^{\prime}$ is relatively weakly compact, then $w\left(M \cdot M^{\prime}\right) \leq\left\|M^{\prime}\right\| w(M)$. 
Remark 1.11 It is worth noticing that every Banach algebra with property $(\mathcal{P})$ is a WCBanach algebra. Also, every commutative Banach algebra with the Dunford-Pettis property has property $(\mathcal{P})$ (see [19]). In particular, $L^{1}(\mu), L^{\infty}(\mu)$, and $C(K)$ have property $(\mathcal{P})$. Recall that a Banach space $X$ is said to have the Dunford-Pettis property (DPP) if for each Banach space $Y$, every weakly compact linear operator $T: X \rightarrow Y$ maps weakly convergent sequences into strongly convergent sequences.

\section{Leray-Schauder-type fixed point theorems in Banach algebras relative to the weak topology}

In this section, we prove some fixed point theorems in Banach algebras relative to the weak topology. Our results are formulated using some Leray-Schauder-type boundary conditions. Before proceeding with the main results, we give a key lemma, which we will employ several times in the sequel.

Lemma 2.1 Let $M$ be a nonempty bounded subset of a Banach algebra $X$, and $A, C: X \rightarrow X$ be Lipschitzian mappings with constants $\alpha_{A}$ and $\alpha_{C}$ such that $\|M\| \alpha_{A}+\alpha_{C}<1$. Then, for every $x \in M$, there is a unique $\tau(x)$ with

$$
\tau(x)=A(\tau(x)) x+C(\tau(x))
$$

Let $\tau_{A, C}: M \rightarrow X$ be the map that assigns to $x \in M$ the value $\tau(x)$. Then:

(i) $\tau_{A, C}(M)$ is bounded.

(ii) $\tau_{A, C}$ is continuous.

(iii) If $X$ is a WC-Banach algebra and if $A$ and $C$ are $w w$-compact, then $\tau_{A, C}$ is ww-compact.

(iv) If $X$ is a Banach algebra with property $(\mathcal{P})$ and if $A$ and $C$ are weakly sequentially continuous, then $\tau_{A, C}$ is weakly sequentially continuous on $M$.

Proof Let $x \in M$ be fixed. The map $\varphi$ that assigns to each $y \in X$ the value $A(y) \cdot x+C(y)$ defines a strict contraction with constant $\|M\| \alpha_{A}+\alpha_{C}$. Indeed, for all $y_{1}, y_{2} \in X$, we have:

$$
\begin{aligned}
\left\|\varphi\left(y_{1}\right)-\varphi\left(y_{2}\right)\right\| & \leq\left\|A y_{1}-A y_{2}\right\|\|x\|+\left\|C y_{1}-C y_{2}\right\| \\
& \leq\|M\| \alpha_{A}\left\|y_{1}-y_{2}\right\|+\alpha_{C}\left\|y_{1}-y_{2}\right\| .
\end{aligned}
$$

Now the contraction mapping principle guarantees that there exists a unique point $\tau(x) \in$ $X$ such that $\varphi(\tau(x))=\tau(x)=A(\tau(x)) x+C(\tau(x))$. Notice that, for all $x \in M$,

$$
\tau(x)=C(\tau(x))+A(\tau(x)) x .
$$

Let $x_{0} \in M$ be fixed. Then, for all $x \in M$, we have

$$
\begin{aligned}
\left\|\tau_{A, C}(x)-\tau_{A, C}\left(x_{0}\right)\right\| & =\left\|\tau(x)-\tau\left(x_{0}\right)\right\| \\
& \leq \alpha_{C}\left\|\tau(x)-\tau\left(x_{0}\right)\right\|+\|M\| \alpha_{A}\left\|\tau(x)-\tau\left(x_{0}\right)\right\|+\left\|A \tau\left(x_{0}\right)\left(x-x_{0}\right)\right\| \\
& \left.\leq\left(\alpha_{C}+\|M\| \alpha_{A}\right) \| \tau(x)-\tau\left(x_{0}\right)\right)\|+\| A \tau\left(x_{0}\right)\|\| x-x_{0} \| .
\end{aligned}
$$


Thus,

$$
\|\tau(x)\| \leq\left\|\tau\left(x_{0}\right)\right\|+\frac{2\|M\|\left\|A \tau\left(x_{0}\right)\right\|}{1-\alpha_{C}-\|M\| \alpha_{A}} .
$$

Consequently, $\tau_{A, C}(M)$ is bounded. Now, we prove that $\tau_{A, C}$ is continuous. For $x_{n} \rightarrow x$, we have

$$
\begin{aligned}
\left\|\tau\left(x_{n}\right)-\tau(x)\right\| & \leq\left\|C \tau\left(x_{n}\right)-C \tau(x)\right\|+\left\|\left(A \tau\left(x_{n}\right)\right) x_{n}-(A \tau(x)) x\right\| \\
& \leq\left\|C \tau\left(x_{n}\right)-C \tau(x)\right\|+\|A \tau(x)\|\left\|x_{n}-x\right\|+\left\|A \tau\left(x_{n}\right)-A \tau(x)\right\|\left\|x_{n}\right\| \\
& \leq \alpha_{C}\left\|\tau\left(x_{n}\right)-\tau(x)\right\|+\alpha_{A}\left\|\tau\left(x_{n}\right)-\tau(x)\right\|\|M\|+\|A \tau(x)\|\left\|x_{n}-x\right\| .
\end{aligned}
$$

Since $\tau_{A, C}(M)$ is bounded, we have lim $\sup _{n}\left\|\tau\left(x_{n}\right)-\tau(x)\right\| \in \mathbb{R}^{+}$, and

$$
\lim \sup _{n}\left\|\tau\left(x_{n}\right)-\tau(x)\right\| \leq \alpha_{C}\left(\lim \sup _{n}\left\|\tau\left(x_{n}\right)-\tau(x)\right\|\right)+\alpha_{A}\left(\lim \sup _{n}\left\|\tau\left(x_{n}\right)-\tau(x)\right\|\right)\|M\| .
$$

Keeping in mind that $\|M\| \alpha_{A}+\alpha_{C}<1$, we deduce that

$$
\lim _{n}\left\|\tau\left(x_{n}\right)-\tau(x)\right\|=0
$$

Now, we prove that $\tau_{A, C}$ is ww-compact. To see this, let $N$ be a weakly compact subset of $M$. Applying the properties of the De Blasi measure of weak noncompactness $w$ and Lemmas 1.8 and 1.10 , we arrive at

$$
w(\tau(N)) \leq w(A(\tau(N)) N)+w(C(\tau(N))) \leq \alpha_{A} w(\tau(N))\|M\|+\alpha_{C} w(\tau(N))
$$

and therefore, $w(\tau(N))=0$. Now, it remains to prove the last assertion. To see this, let $\left(x_{n}\right)$ be a sequence of $M$ that converges weakly to some $x \in M$. Since $\tau_{A, C}$ is ww-compact, there is a subsequence $\left(x_{n_{k}}\right)$ of $\left(x_{n}\right)$ such that the sequence $\tau\left(x_{n_{k}}\right)$ converges weakly to some $z$. Taking into account that $\tau\left(x_{n_{k}}\right)=A \tau\left(x_{n_{k}}\right) x_{n_{k}}+C \tau\left(x_{n_{k}}\right)$ and the fact that $X$ satisfies condition $(\mathcal{P})$, the weak sequential continuity of $A$ and $C$ yields

$$
z=A z x+C z .
$$

Thus, $z=\tau(x)$. Consequently,

$$
\tau\left(x_{n_{k}}\right) \rightarrow z=\tau(x) .
$$

Now we show that

$$
\tau\left(x_{n}\right) \rightarrow \tau(x) .
$$

Suppose the contrary. Then there exists a weak neighborhood $N^{w}$ of $\tau(x)$ and a subsequence $\left(x_{n_{j}}\right)$ of $\left(x_{n}\right)$ such that $\tau\left(x_{n_{j}}\right) \notin N^{w}$ for all $j \geq 1$. Since $\left(x_{n_{j}}\right)$ converges weakly to $x$, then arguing as before, we may extract a subsequence $\left(x_{n_{j_{k}}}\right)$ of $\left(x_{n_{j}}\right)$ such that $\tau\left(x_{n_{j_{k}}}\right) \rightarrow \tau(x)$. 
This is not possible since $\tau\left(x_{n_{j_{k}}}\right) \notin N^{w}$ for all $k \geq 1$. As a result, $\tau_{A, C}$ is weakly sequentially continuous.

Now, we establish our first fixed point theorem under Leray-Schauder boundary conditions.

Theorem 2.2 Let X be a Banach algebra satisfying property $(\mathcal{P})$, and let $\psi$ be a nonsingular measure of weak noncompactness on $X$. Let $\Omega$ be a nonempty closed and convex subset of $X$, and $U \subset \Omega$ be a weakly open set (with respect to the weak topology of $\Omega$ ) such that $0 \in U$. Let $A, C: X \rightarrow X$ and $B: \overline{U^{w}} \rightarrow X$ be weakly sequentially continuous operators satisfying the following conditions:

(i) $B\left(\overline{U^{w}}\right)$ is bounded with bound $Q=\left\|B\left(\overline{U^{w}}\right)\right\|$,

(ii) $A$ and $C$ are Lipschitzian mappings with constants $\alpha_{A}$ and $\alpha_{C}$ such that $Q \alpha_{A}+\alpha_{C}<1$,

(iii) $T:=\tau_{A, C} B: \overline{U^{w}} \rightarrow X$ is $\psi$-condensing.

Then, either

(A1) there exists $u \in \overline{U^{w}}$ such that $\mathrm{AuBu}+\mathrm{Cu}=u$, or

(A2) there exists $u \in \partial_{\Omega} U$ such that

$$
\lambda A\left(\frac{u}{\lambda}\right) B u+\lambda C\left(\frac{u}{\lambda}\right)=u
$$

for some $\lambda \in(0,1)$, where $\partial_{\Omega} U$ is the weak boundary of $U$ in $\Omega$.

Proof First, notice that the equation $\lambda A\left(\frac{u}{\lambda}\right) B u+\lambda C\left(\frac{u}{\lambda}\right)=u$ is equivalent to $\lambda T(u)=u$. Keeping in mind that $B$ is weakly sequentially continuous and $B\left(\overline{U^{w}}\right)$ is bounded and invoking Lemma 2.1, we infer that $T$ is weakly sequentially continuous and $T\left(\overline{U^{w}}\right)=$ $\tau_{A, C} B\left(\overline{U^{w}}\right)$ is bounded. Now, suppose that $(A 2)$ does not occur and $T$ has no fixed point in $\partial_{\Omega} U$ (otherwise, we are finished since (A1) occurs). Then for all $x$ in $\partial_{\Omega} U$ and for all $\lambda$ in $[0,1]$, we have

$$
\lambda T(x) \neq x .
$$

Let

$$
\Delta=\left\{x \in \overline{U^{w}}, \lambda T(x)=x \text { for some } \lambda \in[0,1]\right\} .
$$

The set $\Delta$ is nonempty since $0 \in \Delta$. From (2.1) it follows that $\Delta \cap \partial_{\Omega} U=\emptyset$. Clearly, $\Delta \subset$ $\overline{c o}(T(\Delta) \cup\{0\})$, and so $\Delta$ is bounded. Since $T$ is $\psi$-condensing, we have

$$
\psi(\Delta) \leq \psi(\overline{c o}(T(\Delta) \cup\{0\}))=\psi(T(\Delta) \cup\{0\})=\psi(T(\Delta)),
$$

and therefore $\Delta$ is relatively weakly compact. Our next task is to show that $\Delta$ is weakly sequentially closed. To do this, let $\left(x_{n}\right)_{n}$ be a sequence in $\Delta$ that converges weakly to some $x$. Notice that for each integer $n \in \mathbb{N}$, there is $\lambda_{n} \in[0,1]$ such that $x_{n}=\lambda_{n} T\left(x_{n}\right)$. By extracting a subsequence, if necessary, we assume that $\left(\lambda_{n}\right)_{n}$ converges to some $\lambda \in[0,1]$. Letting $n \rightarrow \infty$, we get $\lambda T x=x$, and so $x \in \Delta$. Now we show that $\Delta$ is weakly closed. To see this, 
let $x \in \overline{U^{w}}$ be weakly adherent to $\Delta$. Since $\overline{\Delta^{w}}$ is weakly compact the Eberlein-Smulian theorem [36], Theorem 8.12.4, p.549, ensures the existence of a sequence $\left(x_{n}\right)_{n} \in \Delta^{\mathbb{N}}$ such that $x_{n} \rightarrow x$. Notice that $x \in \Delta$ since $\Delta$ is weakly sequentially closed. Keeping in mind that $\Delta \cap \partial_{\Omega} U=\emptyset, \Delta$ is weakly compact, and $\partial_{\Omega} U$ is weakly closed since $X$ endowed with the weak topology is a locally convex space (and thus a Tychonoff space), the Urysohn theorem for the weak topology [37] guarantees the existence of a weakly continuous mapping $\varphi: X \rightarrow[0,1]$ such that

$$
\begin{cases}\varphi(x)=1 & \text { for } x \in \Delta, \\ \varphi(x)=0 & \text { for } x \in \partial_{\Omega} U .\end{cases}
$$

We define the mapping $T_{1}: X \longrightarrow X$ by

$$
T_{1}(x)= \begin{cases}\varphi(x) T(x), & x \in \overline{U^{w}}, \\ 0, & x \in X \backslash \overline{U^{w}} .\end{cases}
$$

Since $T$ is weakly sequentially continuous, so is $T_{1}$. Furthermore, we readily check that

$$
T_{1}(X) \subset \overline{c o}\left(T\left(\overline{U^{w}}\right) \cup\{0\}\right)
$$

Let $C=\overline{c o}\left(T\left(\overline{U^{w}}\right) \cup\{0\}\right)$. Clearly, $C$ is a convex closed subset of $X$, and $T_{1}(C) \subset T_{1}(X) \subset C$. Since $T\left(\overline{U^{w}}\right)$ is bounded, so is $T_{1}(C)$.

We consider the set

$$
L=\left\{M \subset C, \overline{c o}(M)=M, 0 \in M \text { and } T_{1}(M) \subset M\right\} .
$$

The set $L$ is nonempty since $C \in L$. Set $N=\bigcap_{M \in L} M$. Clearly, $N$ is a closed convex subset of $C$, and $T_{1}(N) \subset N$. Hence, $N \in L$. Clearly, $\overline{c o}\left(T_{1}(N) \cup\{0\}\right) \subset N$, and so

$$
T_{1}\left(\overline{c o}\left(T_{1}(N) \cup\{0\}\right)\right) \subset T_{1}(N) \subset \overline{c o}\left(T_{1}(N) \cup\{0\}\right) .
$$

Hence, $\overline{c o}\left(T_{1}(N) \cup\{0\}\right) \in L$, and therefore $N \subset \overline{c o}\left(T_{1}(N) \cup\{0\}\right)$. Thus,

$$
N=\overline{c o}\left(T_{1}(N) \cup\{0\}\right) \subset \overline{c o}(T(N) \cup\{0\}) .
$$

Using the properties of the measure of weak noncompactness, we get

$$
\psi(N)=\psi\left(\overline{c o}\left(T_{1}(N) \cup\{0\}\right)\right) \leq \psi(\overline{c o}(T(N) \cup\{0\}))=\psi(T(N)) .
$$

Since $T$ is $\psi$-condensing and $N$ is closed, we deduce that $N$ is weakly compact. The ArinoGautier-Penot fixed point theorem [38] implies that there exists $u \in N$ such that $T_{1}(u)=u$. Now $u \in \overline{U^{w}}$ since $0 \in \overline{U^{w}}$. Consequently, $\varphi(u) T(u)=u$, and so $u \in \Delta$. This implies $\varphi(u)=$ 1 , and so $T(u)=u$. Thus, $A u B u+C u=u$.

Remark 2.3 The statement of Theorem 2.2 remains true if we replace ' $A$ and $C$ Lipschitzian' with ' $A$ and $C \mathcal{D}$-Lipschitzian, provided that $\tau_{A, C} B\left(\overline{U^{w}}\right)$ is bounded. We emphasize that Theorem 2.2 improves [20], Theorem 3.1. In contrast to [20], the operator $A$ in Theorem 2.2 needs not be regular. 
Now, we present some important corollaries of Theorem 2.2, which extend and encompass several well-known results in the literature. Firstly, note that hypothesis (iii) in Theorem 2.2 may be replaced with (iii'): $T\left(\overline{U^{w}}\right)=\tau_{A, C} B\left(\overline{U^{w}}\right)$ is relatively weakly compact or (iii"): $T$ maps bounded sets into relatively weakly compact sets. From an application point of view, it is convenient to require the weak compactness on $B$ instead of $\tau_{A, C} B$. We may therefore state the following result.

Corollary 2.4 Let $X$ be a Banach algebra satisfying property $(\mathcal{P})$. Let $\Omega$ be a nonempty closed and convex subset of $X$, and $U$ be a weakly open subset of $\Omega$ (with respect to the weak topology of $\Omega$ ) such that $0 \in U$. Let $A, C: X \rightarrow X$ and $B: \overline{U^{w}} \rightarrow X$ be weakly sequentially continuous operators satisfying the following conditions:

(i) $B\left(\overline{U^{w}}\right)$ is relatively weakly compact,

(ii) $A$ and $C$ are Lipschitzian mappings with constants $\alpha_{A}$ and $\alpha_{C}$ such that $Q \alpha_{A}+\alpha_{C}<1$, where $Q=\left\|B\left(\overline{U^{w}}\right)\right\|$.

Then, either

(A1) there exists $u \in \overline{U^{w}}$ such that $A u B u+C u=u$, or

(A2) there exist $u \in \partial_{\Omega} U$ and $\lambda \in(0,1)$ such that $\lambda A\left(\frac{u}{\lambda}\right) B u+\lambda C\left(\frac{u}{\lambda}\right)=u$.

Proof It suffices to show that $T:=\tau_{A, C} B$ maps bounded sets of $\overline{U^{w}}$ into relatively weakly compact sets of $X$. To see this, let $M$ be a bounded subset of $\overline{U^{w}}$. Clearly,

$$
T(M) \subset C(T(M))+A(T(M)) B(M)
$$

Let $w$ be the De Blasi measure of weak noncompactness. Referring to Lemmas 1.8 and 1.10, we see that

$$
\begin{aligned}
w(T(M)) & \leq w((C T(M))+A T(M) B(M)) \\
& \leq \psi(C T(M)))+w(A T(M) B(M)) \\
& \leq \alpha_{C} w(T(M))+Q \alpha_{A} w(T(M)) \quad(\text { since } B(M) \text { is relatively weakly, compact }) \\
& \leq\left(\alpha_{C}+Q \alpha_{A}\right) w(T(M)) .
\end{aligned}
$$

Thus, $w(T(M))=0$, and therefore $T(M)$ is relatively weakly compact. The result follows from Theorem 2.2 .

\section{Remark 2.5}

(i) Corollary 2.4 is an analogue of [14], Theorem 2.4, for the weak topology and extends [20], Theorem 3.4.

(ii) In Corollary 2.4, condition (i) may be replaced with (i'): ' $B$ maps bounded sets into relatively weakly compact sets, and $B\left(\overline{U^{w}}\right)$ is bounded.' In this case, if we take $A=1_{X}$, then we obtain the Krasnosel'skii-Leray-Schauder-type fixed point theorem for the weak topology [39], Theorem 2.1.

(iii) If we assume that $\overline{U^{w}}$ is a weakly compact subset of $\Omega$, then hypothesis (iii) in Theorem 2.2 is redundant.

If we take $C=0$ in Theorem 2.2 , then we get the following result. 
Corollary 2.6 Let X be a Banach algebra satisfying property $(\mathcal{P})$, and $\psi$ be a nonsingular measure of weak noncompactness on $X$. Let $\Omega$ be a nonempty closed and convex subset of $X$, and $U \subset \Omega$ be a weakly open set (with respect to the weak topology of $\Omega$ ) such that $0 \in U$. Let $A: X \rightarrow X$ and $B: \overline{U^{w}} \rightarrow X$ be weakly sequentially continuous operators satisfying the following conditions:

(i) $B\left(\overline{U^{w}}\right)$ is bounded with bound $Q=\left\|B\left(\overline{U^{w}}\right)\right\|$,

(ii) $A$ is Lipschitzian mapping with constant $\alpha_{A}$ such that $Q \alpha_{A}<1$,

(iii) $T:=\tau_{A, 0} B: \overline{U^{w}} \rightarrow X$ is a $\psi$-condensing mapping.

Then, either

(A1) there exists $u \in \overline{U^{w}}$ such that $A u B u=u$, or

(A2) there exist $u \in \partial_{\Omega} U$ and $\lambda \in(0,1)$ such that $\lambda A\left(\frac{u}{\lambda}\right) B u=u$.

We describe now an important special case where $C \equiv z_{0} \in X$. We will obtain a sharpening of [20], Theorem 3.2, and a weak Leray-Schauder version of [8], Proposition 2.

Corollary 2.7 Let X be a Banach algebra satisfying property $(\mathcal{P})$, and $\psi$ be a nonsingular measure of weak noncompactness on X. Let $\Omega$ be a nonempty closed and convex subset of $X$, and $U \subset \Omega$ be a weakly open set (with respect to the weak topology of $\Omega$ ) such that $0 \in U$. Let $A: X \rightarrow X$ and $B: \overline{U^{w}} \rightarrow X$ be two weakly sequentially continuous operators. Assume that

(i) A maps bounded sets into bounded sets and there exists a constant $\lambda \geq 0$ such that $\psi(A(M)) \leq \lambda \psi(M)$ for every bounded subset $M$ of $X$, and

(ii) $B$ maps bounded sets into relatively weakly compact sets, $B\left(\overline{U^{w}}\right)$ is bounded, and $\lambda \cdot \sup _{z \in \overline{U^{w}}}\|B(z)\|<1$.

Then, either

(A1) there exists $z \in \overline{U^{w}}$ such that $z_{0}+A z B z=z$, or

(A2) there exist $z \in \partial_{\Omega} U$ and $\lambda \in(0,1)$ such that

$$
\lambda z_{0}+\lambda A\left(\frac{z}{\lambda}\right) B z=z
$$

Proof The reasoning in the proof of Corollary 2.4 yields that $T=\tau_{A, C} B$ is $\psi$-condensing, where $C \equiv z_{0}$. The result follows from Theorem 2.2.

Remark 2.8 If we take $A x=x$ ( $A$ is not regular), in Corollary 2.7, we obtain a weak LeraySchauder version of the Leggett result [7], Theorem 2.

Corollary 2.9 Let $X$ be a Banach algebra satisfying property $(\mathcal{P})$, and let $\psi$ be a nonsingular measure of weak noncompactness on $X$. Let $\Omega$ be a nonempty closed and convex subset of $X$, and $U \subset \Omega$ be a weakly open set (with respect to the weak topology of $\Omega$ ) such that $0 \in U$. Let $A, C: X \rightarrow X$ and $B: \overline{U^{w}} \rightarrow X$ be weakly sequentially continuous operators satisfying conditions (i), (ii), (iii) of Theorem 2.2.

In addition, assume that

$$
\lambda A\left(\frac{u}{\lambda}\right) B u+\lambda C\left(\frac{u}{\lambda}\right) \neq u
$$

for all $\lambda$ in $(0,1)$ and $u$ in $\partial_{\Omega} U$. Then, the set of fixed points of $T$ in $\overline{U^{w}}$ is nonempty and weakly compact. 
Proof Let

$$
\Delta=\left\{x \in \overline{U^{w}}, T(x)=x\right\} .
$$

By Theorem 2.2 we see that $\Delta \neq \emptyset$. Notice that $\Delta \subset T(\Delta)$ implies that $\Delta$ is bounded and

$$
\psi(\Delta) \leq \psi(\overline{c o}(T(\Delta) \cup\{0\})=\psi(T(\Delta)) .
$$

Since $T$ is $\psi$-condensing, $\psi(\Delta)=0$, and therefore $\Delta$ is relatively weakly compact. The reasoning in the proof of Theorem 2.2 shows that $\Delta$ is weakly closed, so $\Delta$ is weakly compact.

Remark 2.10 If we assume that

$$
\|(I-C) x\|>Q\|A x\|
$$

for all $x \in \frac{1}{\lambda} \partial_{\Omega} U$ and $\lambda \in(0,1)$, then, the boundary condition (2.5) is satisfied. Indeed, let $x \in \partial_{\Omega} U$ and $\lambda \in(0,1)$. Suppose that $\lambda A\left(\frac{x}{\lambda}\right) B x+\lambda C\left(\frac{x}{\lambda}\right)=x$. Then $\left\|\frac{x}{\lambda}-C \frac{x}{\lambda}\right\|=\left\|A\left(\frac{x}{\lambda}\right) B x\right\| \leq$ $Q\left\|A\left(\frac{x}{\lambda}\right)\right\|$, which contradicts (2.6).

In many practical situations, the weak (sequential) continuity is not easy to be checked or even not satisfied. So, we will interchange this condition by the ws-compactness. Our approach combines the advantages of the strong topology (i.e., the involved mappings will be continuous) with the advantages of the weak topology (i.e., the maps will be weakly compact). This enlarges considerably the applicability of our fixed point theorems. In analogy to what we have done in beginning of Section 2, we will try to develop a parallel fixed point theory for ws-compact operators under Leray-Schauder-type boundary conditions.

Theorem 2.11 Let $X$ be a Banach algebra, and $\psi$ be a nonsingular measure of weak noncompactness on $X$. Let $\Omega$ be a nonempty closed and convex subset of $X$, and $U \subset \Omega$ be an open set (with respect to the topology of $\Omega$ ) such that $0 \in U$. Let $A, C: X \longrightarrow X$ and $B: \bar{U} \longrightarrow X$ be three operators satisfying the following conditions:

(i) $B$ is ws-compact, and the set $B(\bar{U})$ is bounded with bound $Q=\|B(\bar{U})\|$,

(ii) $A$ and $C$ are Lipschitzian mappings with constants $\alpha_{A}$ and $\alpha_{C}$ such that

$$
Q \alpha_{A}+\alpha_{C}<1
$$

(iii) $T:=\tau_{A, C} B: \bar{U} \rightarrow X$ is $\psi$-condensing.

Then, either

(A1) there exists $u \in \bar{U}$ such that $A u B u+C u=u$, or

(A2) there exist $u \in \partial_{\Omega} U$ and $\lambda \in(0,1)$ such that $\lambda A\left(\frac{u}{\lambda}\right) B u+\lambda C\left(\frac{u}{\lambda}\right)=u$.

Proof Referring to Lemma 2.1, we see that the operator $T=\tau_{A, C} B: \bar{U} \longrightarrow X$ is well defined and continuous. Notice also that since $B$ is ws-compact, $T$ is ws-compact. Now, suppose that (A2) does not occur and $T$ has no fixed point in $\partial_{\Omega} U$ (otherwise, we are finished since (A1) occurs). Then, for all $x$ in $\partial_{\Omega} U$ and for all $\lambda$ in $[0,1]$, we have that 
Let

$$
\Delta=\left\{x \in \overline{U^{w}}, \lambda T(x)=x \text { for some } \lambda \in[0,1]\right\} .
$$

The set $\Delta$ is nonempty since $0 \in \Delta$. In addition, the continuity of $T$ implies that $\Delta$ is closed. From (2.7) we know that

$$
\Delta \cap \partial_{\Omega} U=\emptyset .
$$

Clearly,

$$
\Delta \subset \overline{c o}(T(\Delta) \cup\{0\})
$$

Applying the properties of $\psi$, we have

$$
\psi(\Delta) \leq \psi(\overline{c o}(T(\Delta) \cup\{0\}))=\psi(T(\Delta) \cup\{0\})=\psi(T(\Delta)) .
$$

Since $T$ is $\psi$-condensing, $\Delta$ is relatively weakly compact. Notice that $\Delta$ is closed implies $\Delta$ is weakly compact. Since $T$ is ws-compact, $T(\Delta)$ is relatively compact. It follows, by using the Mazur theorem, that $\overline{c o}(T(\Delta) \cup\{0\})$ is compact. Now, (2.10), together with the fact that $\Delta$ is closed, implies that $\Delta$ is compact. By Urysohn's lemma [37] there exists a continuous function $\varphi: \bar{U} \longrightarrow[0,1]$ with $\varphi(\Delta)=1$ and $\varphi\left(\partial_{\Omega} U\right)=0$. Let

$$
F(x)= \begin{cases}\varphi(x) T(x), & x \in \bar{U}, \\ 0, & x \in X \backslash \bar{U} .\end{cases}
$$

Clearly, $F$ is a continuous mapping, and

$$
F(X) \subset \overline{c o}(T(\bar{U}) \cup\{0\}) .
$$

Invoking Lemma 2.1, we infer that $T(\bar{U})$ is bounded. Now, let $K=\overline{c o}(T(\bar{U}) \cup\{0\})$. Obviously, $K$ is a closed convex subset of $X$, and $F(K) \subset F(X) \subset K$. Since $T(\bar{U})$ is bounded, so is $F(K)$. Consider

$$
L=\{M \subset C, \overline{c o}(M)=M, 0 \in M \text { and } F(M) \subset M\} .
$$

The reasoning in the proof of Theorem 2.2 yields that $N=\bigcap_{M \in L} M$ is weakly compact. Since $T$ is ws-compact, $T(N)$ is relatively compact. The Mazur theorem guarantees that $\overline{c o}(T(N) \cup\{0\})$ is compact. This implies that $N$ is compact since $N \subset \overline{c o}(T(N) \cup\{0\})$. Now, the Schauder fixed point theorem implies that there is $u \in N$ such that $F(u)=u$. Now $u \in \bar{U}$ since $0 \in \bar{U}$. Consequently, $\varphi(u) T(u)=u$, and so $u \in \Delta$. This implies $\varphi(u)=1$, and so $T(u)=u$. Thus, $A u B u+C u=u$.

Remark 2.12 We should mention that property $(\mathcal{P})$ is essential in the proof of Theorem 2.2 as far as the weak sequential continuity is concerned. This is not the case in Theorem 2.11 since the involved mappings are continuous (for the norm topology) and the algebra multiplication is always continuous. 
As a consequence of Theorem 2.11, we get the following corollaries and related results.

Corollary 2.13 Let $X$ be a Banach algebra. Let $\Omega$ be a nonempty closed and convex subset of $X$, and $U \subset \Omega$ be an open set (with respect to the topology of $\Omega$ ) such that $0 \in U$. Let $A, C: X \longrightarrow X$ and $B: \bar{U} \longrightarrow X$ be three operators satisfying the following conditions:

(i) $B$ is ws-compact, and the set $B(\bar{U})$ is bounded with bound $Q=\|B(\bar{U})\|$,

(ii) $A$ and $C$ are Lipschitzian mappings with constants $\alpha_{A}$ and $\alpha_{C}$ such that

$$
Q \alpha_{A}+\alpha_{C}<1
$$

(iii) $T:=\tau_{A, C} B: \bar{U} \rightarrow X$ is weakly compact.

Then, either

(A1) there exists $u \in \bar{U}$ such that $\mathrm{AuBu}+\mathrm{Cu}=u$, or

(A2) there exist $u \in \partial_{\Omega} U$ and $\lambda \in(0,1)$ such that $\lambda A\left(\frac{u}{\lambda}\right) B u+\lambda C\left(\frac{u}{\lambda}\right)=u$.

Remark 2.14 In Corollary 2.13, hypothesis (iii) may be replaced with (iii'): ' $T$ maps bounded sets into relatively weakly compact sets.'

Corollary 2.15 Let X be a WC-Banach algebra. Let $\Omega$ be a nonempty closed and convex subset of $X$, and $U \subset \Omega$ be an open set (with respect to the topology of $\Omega$ ) such that $0 \in U$. Let $A, C: X \longrightarrow X$ and $B: \bar{U} \longrightarrow X$ be three operators satisfying the following conditions:

(i) $B$ is ws-compact, $B$ maps bounded sets into relatively weakly compact sets, and $B(\bar{U})$ is bounded with bound $Q$,

(ii) A and C are ww-compact Lipschitzian mappings with constants $\alpha_{A}$ and $\alpha_{C}$ such that

$$
Q \alpha_{A}+\alpha_{C}<1
$$

Then, either

(A1) there exists $u \in \bar{U}$ such that $A u B u+C u=u$, or

(A2) there exist $u \in \partial_{\Omega} U$ and $\lambda \in(0,1)$ such that $\lambda A\left(\frac{u}{\lambda}\right) B u+\lambda C\left(\frac{u}{\lambda}\right)=u$.

Proof According to Corollary 2.13, we have only to show that $T$ maps bounded sets into relatively weakly compact sets. To see this, let $M$ a bounded subset of $\bar{U}$. From our hypotheses we know that $B(M)$ is relatively weakly compact. Notice $\tau_{A, C}$ is $w w$-compact implies that $T(M)=\tau_{A, C}(B(M))$ is relatively weakly compact. This achieves the proof.

\section{Remark 2.16}

(1) In Corollary 2.15, hypothesis (i) can be replaced with (i'): ' $B$ is ws-compact, and $B(\bar{U})$ is relatively weakly compact.'

(2) Corollary 2.15 is a weak version of [14], Theorem 2.4.

Considering the particular case $C \equiv 0$, we get the following result.

Corollary 2.17 Let X be a Banach algebra, and $\psi$ be a nonsingular measure of weak noncompactness on $X$. Let $\Omega$ be a nonempty closed and convex subset of $X$, and $U \subset \Omega$ be an open set (with respect to the topology of $\Omega$ ) such that $0 \in U$. Let $A: X \longrightarrow X$ and $B: \bar{U} \longrightarrow X$ are continuous operators satisfying the following conditions:

(i) B is ws-compact, and $B(\bar{U})$ is bounded with bound $Q=\|B(\bar{U})\|$, 
(ii) $A$ is a Lipschitzian mapping with constant $\alpha_{A}$ such that $Q \alpha_{A}<1$,

(iii) $T:=\tau_{A, 0} B: \bar{U} \rightarrow X$ is $\psi$-condensing.

Then, either

(A1) there exists $u \in \bar{U}$ such that $A u B u=u$, or

(A2) there exist $u \in \partial_{\Omega} U$ and $\lambda \in(0,1)$ such that $\lambda A\left(\frac{u}{\lambda}\right) B u=u$.

Remark 2.18 The results of Section 2 remain true if we replace $A$ and $C$ 'Lipschitzian' with 'D-Lipschitzian, provided that $\tau_{A, C} B\left(\overline{U^{w}}\right)$ is bounded.

\section{Application}

As pointed out in the Introduction, quadratic integral equations occur in a large number of 'real-life' problems. In order to illustrate our abstract mathematical results, we will devote the present section to study of the existence of continuous solutions to the following abstract nonlinear quadratic integral equation:

$$
x(t)=k\left(t, x(\mu(t))+A x(t)\left(q(t)+\int_{0}^{\sigma(t)} g(s, x(\eta(s))) d s\right), \quad t \in J=[0,1]\right.
$$

where $X$ is a real Banach algebra satisfying condition $(\mathcal{P})$. As usual, we will denote by $E=C([0,1], X)$ the Banach space of all $X$-valued continuous functions defined on $[0,1]$, endowed with its standard norm $\|x\|=\sup _{t \in[0,1]}\|x(t)\|$. Notice that $E$ is a Banach algebra that satisfies condition $(\mathcal{P})$. The operator $A$ maps $E$ into itself. The functions $q: J \longrightarrow$ $X, g, k: J \times X \longrightarrow X$, and $\mu, \sigma, \eta: J \longrightarrow J$ satisfy conditions to be specified later. The integral in (3.1) is understood to be the Pettis integral (see [40] for more details), and solutions to (3.1) will be sought in $E$. The solvability of equation (3.1) is motivated by the previous papers $[14-17,19,20]$. Equation (3.1) will be studied under the following assumptions:

(H1) (i) The functions $\mu, \sigma, \eta: J \longrightarrow J$ are continuous,

(ii) the function $q: J \longrightarrow X$ is continuous. Let $Q_{1}=\sup _{t \in J}\|q(t)\|$,

(H2) (i) for all $t \in[0,1], k(t, \cdot): X \longrightarrow X$ is weakly sequentially continuous,

(ii) for each $x \in X, k(\cdot, x): J \longrightarrow X$ is continuous,

(iii) there is a continuous function $\delta: J \longrightarrow \mathbb{R}^{+}$with bound $\Delta=\|\delta\|_{\infty}$ such that $\|k(t, x)-k(t, y)\| \leq \delta(t)\|x-y\|$ for all $x, y \in X$ and $t \in[0,1]$,

(H3) the operator $A: E \longrightarrow E$ satisfies

(i) there exists a continuous function $\gamma: J \longrightarrow \mathbb{R}^{+}$with bound $\Gamma=\|\gamma\|_{\infty}$ such that, for all $x, y \in E$ and $t \in[0,1]$,

$$
\|A x(t)-A y(t)\| \leq \gamma(t)\|x(t)-y(t)\|
$$

(ii) $A$ is weakly sequentially continuous on $E$,

(iii) $A$ is weakly compact, and $M_{0}=\sup _{x \in E}\|A x\|$,

(H4) (i) for each continuous $x:[0,1] \longrightarrow X$, the function $s \mapsto g(s, x(s))$ is weakly measurable on $[0,1]$, and for almost every $t \in[0,1]$, the map $x \mapsto g(t, x)$ is weakly sequentially continuous,

(ii) there are a function $\phi \in L^{1}\left([0,1], \mathbb{R}^{+}\right)$and a continuous nondecreasing function $\theta: \mathbb{R}^{+} \longrightarrow \mathbb{R}^{+}$such that

$$
\|g(s, x)\| \leq \phi(s) \theta(\|x\|) \quad \text { for a.e. } s \in[0,1]
$$

and all $x$ in $X$, 
(iii) there is a constant $\tau \geq 0$ such that $\tau \frac{M_{0}}{1-k_{C}}<1$ and

$$
w(g([0,1] \times V)) \leq \tau w(V)
$$

for any bounded subset $V$ of $X$,

(H5) there is $r>0$ such that $Q \Gamma+\Delta<1$, where

$$
Q=Q_{1}+\theta(r) \int_{0}^{1} \phi(s) d s
$$

Let $r>0$ satisfy (H5), and let $\Omega$ be any closed convex equicontinuous subset of $B(0, r)$ with $0 \in \Omega$. Let $U$ be a weakly open subset of $\Omega$ such that $0 \in U$. Notice that (3.1) is equivalent to the operator equation

$$
A x B x+C x=x
$$

where the operators $C$ and $B$ are defined by

$$
\begin{aligned}
& B x(t)=q(t)+\int_{0}^{\sigma(t)} g(s, x(\eta(s))) d s, \quad t \in J, \\
& C x(t)=k(t, x(\mu(t)) .
\end{aligned}
$$

Now, we are ready to state the main result of this section.

Theorem 3.1 Let $X$ be a Banach algebra satisfying condition $(\mathcal{P})$ and suppose that assumptions (H1)-(H5) hold.

In addition, assume that

$$
Q\left\|A\left(\frac{1}{\lambda} x\right)(t)\right\|<\left\|\frac{1}{\lambda} x(0)-k\left(0, \frac{1}{\lambda} x(\eta(0))\right)\right\|
$$

for all $\lambda \in(0,1), t \in[0,1]$, and $x \in \partial_{\Omega} U$.

Then equation (3.1) has at least one solution in $E$.

To prove Theorem 3.1, we need the following result.

Lemma 3.2 ([41], p.36) Let $K$ be a compact Hausdorff space, and X be a Banach space. Let $\left(f_{n}\right)_{n}$ be a bounded sequence in $C(K, X)$, and $f \in C(K, X)$. Then $\left(f_{n}\right)_{n}$ is weakly convergent to $f$ if and only if $\left(f_{n}(t)\right)_{n}$ is weakly convergent to $f(t)$ for each $t \in K$.

Proof We show that the operators $A, B$, and $C$ satisfy all conditions of Corollary 2.9. This will be achieved in five steps.

Step 1: We show that the operators $A$ and $C$ make sense and are weakly sequentially continuous. In view of hypothesis (H3)(ii), $A$ is weakly sequentially continuous. Now, we show that the mapping $C$ is well defined. To this end, let $x \in E$ and let $\left(t_{n}\right) \in J^{\mathbb{N}}$ be such 
that $t_{n} \longrightarrow t \in J$. Thus,

$$
\begin{aligned}
\left\|C x\left(t_{n}\right)-C x(t)\right\| & =\left\|k\left(t_{n}, x\left(\mu\left(t_{n}\right)\right)\right)-k(t, x(\mu(t)))\right\| \\
& \leq\left\|k\left(t_{n}, x\left(\mu\left(t_{n}\right)\right)\right)-k\left(t_{n}, x(\mu(t))\right)\right\|+\| k\left(t_{n}, x(\mu(t))\right)-k(t, x(\mu(t)) \| \\
& \leq \delta\left(t_{n}\right)\left\|x\left(\mu\left(t_{n}\right)\right)-x(\mu(t))\right\|+\left\|k\left(t_{n}, x(\mu(t))\right)-k(t, x(\mu(t)))\right\| \\
& \leq \Delta\left\|x\left(\mu\left(t_{n}\right)\right)-x(\mu(t))\right\|+\left\|k\left(t_{n}, x(\mu(t))\right)-k(t, x(\mu(t)))\right\| .
\end{aligned}
$$

Since $k(\cdot, x)$ is continuous (from (H2)(ii)) and $\mu$ is continuous, it follows that $\| C x\left(t_{n}\right)-$ $C x(t) \| \longrightarrow 0$. Accordingly, $C x \in E$. Now, we show that $C$ is weakly sequentially continuous. To see this, let $\left(x_{n}\right)_{n}$ be a sequence in $E$ such that $x_{n} \rightarrow x$ for some $x \in E$. Then $\left(x_{n}\right)_{n}$ is bounded in $E$. Also, for each $t \in[0,1], x_{n}(\mu(t)) \rightarrow x(\mu(t))$ (by Lemma 3.2). Hence, $C x_{n}(t)=$ $k\left(t, x_{n}(t)\right) \rightarrow k(t, x(t))=C x(t)($ by $(\mathrm{H} 2)(\mathrm{i}))$. Using again Lemma 3.2, we infer that $C x_{n} \rightarrow C x$.

Step 2: Now, we show that $A$ and $C$ are Lipschitzian functions. To do this, let $x, y \in E$. From assumption (H3)(i) it follows that

$$
\|A(x)(t)-A(y)(t)\| \leq \gamma(t)\|x(t)-y(t)\| \leq \Gamma\|x-y\|
$$

for each $t \in J$. As a result,

$$
\|A x-A y\| \leq \Gamma\|x-y\|
$$

In virtue of assumption (H2)(iii), the reasoning above yields that $C$ is Lipschitzian with constant $\Delta$.

Step 3: Now, we illustrate that $B$ maps $\Omega$ into $E$ and is weakly sequentially continuous. To perform this, let $x \in \Omega$ and $u, v \in[0,1]$. Without loss of generality, we may assume that $|B x(v)-B x(u)| \neq 0$. Using the Hahn-Banach theorem, we deduce that there exists $h \in X^{\star}$ such that $h(B x(v)-B x(u))=\|B x(v)-B x(u)\|$ and $\|h\|=1$. Hence,

$$
\begin{aligned}
\|B x(v)-B x(u)\| & =h(B x(v)-B x(u)) \\
& =h(q(v)-q(u))+\int_{\sigma(u)}^{\sigma(v)} h(g(s, x(\eta(s)))) d s \\
& \leq\|q(v)-q(u)\|+\theta(r) \int_{\sigma(u)}^{\sigma(v)} \phi(s) d s .
\end{aligned}
$$

Accordingly, $B x \in E$. Furthermore, the uniform continuity of $q$ and $\sigma$ on the compact $[0,1]$ implies that $(B(\Omega))$ is an equicontinuous family of functions. On the other hand, we show that $B(\Omega)$ is bounded. In fact, fix $x \in \Omega$ and take $t \in[0,1]$. Without loss of generality, we may assume that $|B x(t)| \neq 0$. Using the Hahn-Banach theorem, we deduce that there exists $h \in X^{\star}$ such that $h(B x(t))=\|B x(t)\|$ and $\|h\|=1$. It follows that

$$
\begin{aligned}
\|B x(t)\| & =h(B x(t)) \\
& =h(q(t))+\int_{0}^{\sigma(t)} h(g(s, x(\eta(s)))) d s \\
& \leq\|q\|+\int_{0}^{1}\|g(s, x(s))\| d s
\end{aligned}
$$




$$
\begin{aligned}
& \leq Q_{1}+\int_{0}^{1} \phi(s) \theta(\|x(\eta(s))\|) d s \\
& \leq Q_{1}+\theta(r) \int_{0}^{1} \phi(s) d s=Q,
\end{aligned}
$$

and thus $B(\Omega)$ is bounded.

Now, we show that $B$ is weakly sequentially continuous on $\Omega$. To see this, let $\left(x_{n}\right)_{n} \in \Omega^{\mathbb{N}}$ and $x \in \Omega$ be such that $x_{n} \rightarrow x$. Then, $\left(x_{n}\right)$ is norm bounded. Applying Lemma 3.2, we get

$$
x_{n}(t) \rightarrow x(t), \quad t \in[0,1] .
$$

Furthermore, for $n$ in $\mathbb{N}$ and $\psi \in X^{\star}$,

$$
\psi\left(B x_{n}(t)\right)=\psi(q(t))+\int_{0}^{\sigma(t)} \psi\left(g\left(s, x_{n}(\eta(s))\right)\right) d s, \quad t \in[0,1] .
$$

From hypotheses (H1)(i) and (H4)(i) it follows that

$$
\psi \circ g\left(s, x_{n}(\eta(s))\right) \longrightarrow \psi \circ g(s, x(\eta(s)))
$$

for each $s \in[0,1]$. The Lebesgue dominated convergence theorem yields

$$
\int_{0}^{\sigma(t)} \psi \circ g\left(s, x_{n}(\eta(s))\right) d s \longrightarrow \int_{0}^{\sigma(t)} \psi \circ g(s, x(\eta(s))) d s .
$$

As a result, $B x_{n}(t) \rightarrow B x(t)$. Applying again Lemma 3.2, we conclude that $B x_{n} \rightarrow B x$.

Step 4: Now, we prove that $T$ is $w$-condensing, where $w$ is the De Blasi measure of weak noncompactness. Indeed, let $S$ be a bounded subset of $\Omega$ such that $w(S)>0$. Notice that, for each $x \in S$, we have $T x=A(T x) B x+C(T x)$. Hence,

$$
T(S) \subset A(T(S)) B(S)+C(T(S))
$$

Thus,

$$
w(T(S)) \leq w(A(T(S)) B(S))+w(C(T(S)))
$$

From Lemma 1.10 (since $A$ is weakly compact) it follows that

$$
w(T(S)) \leq\|A(T(S))\| w(B(S))+w(C(T(S))) \leq M_{0} w(B(S))+k_{C} w(T(S)) .
$$

Consequently,

$$
w(T(S)) \leq \frac{M_{0}}{1-k_{C}} w(B(S)) .
$$

On the other hand, for each $t \in[0,1]$, we have

$$
\begin{aligned}
w(B(S)(t)) & \leq w\left(\left\{\int_{0}^{\sigma(t)} g(s, x(\eta(s))) d s: x \in S\right\}\right) \\
& \leq w(\sigma(t) \overline{c o}\{g(s, x(\eta(s))): x \in S, s \in[0,1]\}) \quad \text { (by Lemma 1.7) }
\end{aligned}
$$




$$
\begin{aligned}
& \leq w(g([0,1] \times S[0,1])) \\
& \leq \tau w(S[0,1])
\end{aligned}
$$

Theorem 1.6 implies (since $S$ is bounded and equicontinuous) that

$$
w(S[0,1]) \leq w(S)
$$

Hence, for each $t \in[0,1]$, we have

$$
w(B(S)(t)) \leq \tau w(S) .
$$

Invoking Theorem 1.6, we obtain (since $B(S)$ is bounded and equicontinuous)

$$
w(B(S)) \leq \tau w(S) .
$$

Consequently,

$$
w(T(S)) \leq \tau \frac{M_{0}}{1-k_{C}} w(S) .
$$

Since $\tau \frac{M_{0}}{1-k_{C}}<1$, we have $w(T(S))<w(S)$.

Step 5: Finally, it remains to show that condition (2.6) of Remark 2.10 is satisfied. Let $\lambda$ in $(0,1)$ and $x \in \partial_{\Omega} U$. The use of property (3.5) implies that, for each $t \in[0,1]$,

$$
\begin{aligned}
Q\left\|A\left(\frac{1}{\lambda} x\right)(t)\right\| & <\left\|\frac{1}{\lambda} x(0)-k\left(0, \frac{1}{\lambda} x(\eta(0))\right)\right\| \\
& \leq\left\|\frac{1}{\lambda} x-C\left(\frac{1}{\lambda} x\right)\right\| .
\end{aligned}
$$

Applying Corollary 2.9, we infer that there is $u \in \Omega$ such that

$$
A u B u+C u=u,
$$

which is in turn a solution of the operator equation (3.1) in $E$.

Remark 3.3 Our results are more general than those obtained in [20]. In [20], $X$ is assumed to be a reflexive Banach algebra, and $A$ is assumed to be quasi-regular. In our considerations, it might be, from technical viewpoint, relatively simple to require $X$ to be reflexive and to use standard arguments based on the weak version of the ArzelàAscoli theorem to obtain existence results under weaker assumptions. Unfortunately, the only known examples of Banach algebras with property $(\mathcal{P})$ are Banach algebras with the Dunford-Pettis property (see [19]). Nevertheless, the Dunford-Pettis property is thought of as the opposite of reflexivity because it is known that a reflexive Banach algebra with the Dunford-Pettis property is necessarily finite-dimensional. For this reason, we may ask whether some infinite-dimensional reflexive Banach algebra could have property $(\mathcal{P})$. 
Authors' contributions

All authors contributed equally and significantly in writing this article. All authors read and approved the final manuscript.

\section{Author details}

'Département de Mathématiques, Faculté des Sciences Semlalia, Université Cadi Ayyad, B. P. 2390, Marrakech, Morocco.

${ }^{2}$ National School of Applied Sciences, Cadi Ayyad University, Marrakesh, Morocco.

\section{Acknowledgements}

The authors express their gratitude to the anonymous referees for their very valuable remarks and improvements.

\section{Received: 20 February 2016 Accepted: 18 August 2016 Published online: 01 September 2016}

\section{References}

1. Chandrasekhar, S: Radiative Transfer. Dover, New York (1960)

2. George, H, Pimbley, JR: Positive solutions of a quadratic integral equation. Arch. Ration. Mech. Anal. 24(2), 107-127 (1967)

3. Gripenberg, G: On some epidemic models. Q. Appl. Math. 39, 317-327 (1981)

4. Kurz, L, Nowosad, P, Saltzberg, BR: On the solution of a quadratic integral equation arising in signal design. J. Franklin Inst. 281, 437-454 (1966)

5. Hu, S, Khavanin, M, Zhuang, W: Integral equations arising in the kinetic theory of gases. Appl. Anal. 34(3-4), 261-266 (1989)

6. Spiga, G, Bowden, RL, Boffi, VC: On the solutions to a class of nonlinear integral equations arising in transport theory. J. Math. Phys. 25, 3444 (1984)

7. Leggett, RW: On certain nonlinear integral equations. J. Math. Anal. Appl. 57, 462-468 (1977)

8. Majorana, A, Marano, SA: Continuous solutions of a nonlinear integral equation on an unbounded domain. J. Integral Equ. Appl. 6(1), 119-128 (1994)

9. Banaś, J, Lecko, M: Fixed points of the product of operators in Banach algebra. Panam. Math. J. 12(2), 101-109 (2002)

10. Dhage, BC: On some variants of Schauder's fixed point principle and applications to nonlinear integral equations. J. Math. Phys. Sci. 25, 603-611 (1988)

11. Dhage, BC: Remarks on two fixed point theorems involving the sum and the product of two operators. Comput. Math. Appl. 46, 1779-1785 (2003)

12. Dhage, BC: A fixed point theorem in Banach algebras involving three operators with applications. Kyungpook Math. J. 44, 145-155 (2004)

13. Dhage, BC: On a fixed point theorem in Banach algebras with applications. Appl. Math. Lett. 18, $273-280$ (2005)

14. Dhage, BC: On some nonlinear alternatives of Leray-Schauder type and functional integral equations. Arch. Math. 42, $11-23(2006)$

15. Banaś, J, Rodriguez, JR, Sadarangani, K: On a class of Urysohn-Stieltjes quadratic integral equations and their applications. J. Comput. Appl. Math. 113(1), 35-50 (2000)

16. Banaś, J, Rodriquez, JR, Sadarangani, K: On a nonlinear quadratic integral equation of Urysohn-Stieltjes type and its applications. Nonlinear Anal., Theory Methods Appl. 47(2), 1175-1186 (2001)

17. Benchohra, M, Darwish, MA: On quadratic integral equations of Urysohn type in Fréchet spaces. Acta Math. Univ. Comen. 79(1), 105-110 (2010)

18. Djebali, S, Hammache, K: Furi-Pera fixed point theorems in Banach algebras with applications. Acta Univ. Palacki. Olomuc., Fac. Rerum Nat., Math. 47, 55-75 (2008)

19. Banaś, J, Taoudi, MA: Fixed points and solutions of operator equations for the weak topology in Banach algebras. Taiwan. J. Math. 18(3), 871-893 (2014)

20. Ali, AA, Ben Amar, A: Measures of weak noncompactness, nonlinear Leray-Schauder alternatives in Banach algebras satisfying condition $(\mathcal{P})$ and an application. Quaest. Math. 39, 319-340 (2015)

21. Ben Amar, A, Chouayekh, S, Jeribi, A: New fixed point theorems in Banach algebras under weak topology features and applications to nonlinear integral equation. J. Funct. Anal. 259(9), 2215-2237 (2010)

22. Banaś, J, Rivero, J: On measures of weak noncompactness. Ann. Mat. Pura Appl. 151, 213-224 (1988)

23. De Blasi, FS: On a property of the unit sphere in Banach spaces. Bull. Math. Soc. Sci. Math. Roum. 21, $259-262$ (1977)

24. Latrach, K, Taoudi, M-A, Zeghal, A: Some fixed point theorems of the Schauder and Krasnoselskii type and application to nonlinear transport equations. J. Differ. Equ. 221(1), 256-271 (2006)

25. Agarwal, RP, O'Regan, D, Taoudi, M-A: Fixed point theorems for ws-compact mappings in Banach spaces. Fixed Point Theory Appl. 2010, Article ID 183596 (2010)

26. Djebali, S, Sahnoun, Z: Nonlinear alternatives of Schauder and Krasnosel'skii types with applications to Hammerstein integral equations in $L^{1}$ spaces. J. Differ. Equ. 249(9), 2061-2075 (2010)

27. Jachymski, J: On Isac's fixed point theorem for selfmaps of a Galerkin cone. Ann. Sci. Math. Qué. 18(2), 169-171 (1994)

28. Latrach, K, Taoudi, M-A: Existence results for a generalized nonlinear Hammerstein equation on $L^{1}$-spaces. Nonlinear Anal. 66, 2325-2333 (2007)

29. Salhi, N, Taoudi, M-A: Existence of integrable solutions of an integral equation of Hammerstein type on an unbounded interval. Mediterr. J. Math. 9(4), 729-739 (2012)

30. Taoudi, M-A: Integrable solutions of a nonlinear functional integral equation on an unbounded interval. Nonlinear Anal. 71, 4131-4136 (2009)

31. Taoudi, M-A, Salhi, N, Ghribi, B: Integrable solutions of a mixed type operator equation. Appl. Math. Comput. 216(4), 1150-1157 (2010)

32. Ezzinbi, K, Taoudi, M-A: Sadovskii-Krasnosel'skii type fixed point theorems in Banach spaces with application to evolution equations. J. Appl. Math. Comput. 49(1-2), 243-260 (2015)

33. O'Regan, D: Operator equations in Banach spaces relative to the weak topology. Arch. Math. 71(2), 123-136 (1998)

34. Cichon, M, Kubiaczyk, I, Sikorska, A: The Henstock-Kurzweil-Pettis integrals and existence theorems for the Cauchy problem. Czechoslov. Math. J. 54(2), 279-289 (2004) 
35. Agarwal, RP, O'Regan, D, Taoudi, M-A: Browder-Krasnoselskii-type fixed point theorems in Banach spaces. Fixed Point Theory Appl. 2010, Article ID 243716 (2010)

36. Edwards, RE: Theory and Applications, Functional Analysis. Holt, Rinehart \& Winston, New York (1965)

37. James, IM: Topological and Uniform Spaces. Springer, New York (1987)

38. Arino, O, Gautier, S, Penot, JP: A fixed point theorem for sequentially continuous mappings with applications to ordinary differential equations. Funkc. Ekvacioj 27, 273-279 (1984)

39. O'Regan, D, Taoudi, MA: Fixed point theorems for the sum of two weakly sequentially continuous mappings. Nonlinear Anal., Theory Methods Appl. 73(2), 283-289 (2010)

40. Pettis, BJ: On integration in vector spaces. Trans. Am. Math. Soc. 44, 277-304 (1938)

41. Dobrakov, I: On representation of linear operators on $C_{0}(T, X)$. Czechoslov. Math. J. 21, 13-30 (1971)

Submit your manuscript to a SpringerOpen ${ }^{\odot}$ journal and benefit from:

$\rightarrow$ Convenient online submission

Rigorous peer review

- Immediate publication on acceptance

- Open access: articles freely available online

- High visibility within the field

- Retaining the copyright to your article

Submit your next manuscript at springeropen.com 